\title{
Spatially resolved measurement of chromatic dispersion and Brillouin shift along single-mode optical fibers
}

\author{
Miguel González Herráez and Luc Thévenaz \\ ${ }^{a}$ Nanophotonics and Metrology Laboratory, École Polytechnique Fédérale de Lausanne, EPFL. \\ Lausanne CH-1015, Switzerland. E-mail: miguel.gonzalez@epfl.ch.
}

\begin{abstract}
We present a new technique for simultaneous measurement of chromatic dispersion and Brillouin shift distribution along conventional, single-mode optical fibers used in telecommunications. It is based on performing Brillouin Optical Time Domain Analysis (BOTDA) over the four-wave mixing generated by two low-power pumps $(<10 \mathrm{~mW})$. Unlike others, this technique can measure any arbitrary value of dispersion. With the use of this technique we have obtained consistent, high-resolution and low-noise measurements for both magnitudes. Our measurements indicate a certain correlation between the spatial distribution of both quantities, although the degree of correlation varies for the different manufacturers tested.
\end{abstract}

Keywords: Chromatic dispersion, Brillouin shift, Brillouin Optical Time Domain Analysis.

\section{INTRODUCTION}

Nonlinear transmission in optical fibers is governed not only by the average (end-to-end) value of chromatic dispersion along the fiber, but also by the local fluctuations of the dispersion coefficient. A precise knowledge of the evolution of chromatic dispersion along the fiber has been shown to be beneficial in recent experiments using massive wavelengthdivision multiplexing (WDM) and dispersion-managed soliton transmission [1]. As a result of this potential benefit, several methods for mapping chromatic dispersion fluctuations in optical fibers have already been presented in the literature [2-4].

This paper presents a new method for simultaneous, position-resolved measurement of chromatic dispersion and Brillouin shift along an optical fiber. This new technique does not only overcome some limitations of previous chromatic dispersion mapping techniques but also might reveal the cause of the chromatic dispersion variations along the fiber by relating them to strain or doping concentration variations. Moreover, our preliminary measurements suggest a correlation between both magnitudes, although this correlation seems to depend on the manufacturing process used.

\section{MEASUREMENT PRINCIPLE}

We consider two pumps $\left(f_{I}<f_{K}\right)$ delivering a few milliwatts $(5-10 \mathrm{~mW})$ of power at the fiber input. By means of a fourwave mixing (FWM) process, a Stokes and an anti-Stokes wave will be generated along the fiber, following energy conservation rules $\left(f_{S}=2 f_{I}-f_{K}, f_{A}=2 f_{K}-f_{I}\right)$. The power of the anti-Stokes wave oscillates along the fiber with a spatial frequency $\Delta \beta=2 \beta\left(f_{K}\right)-\beta\left(f_{i}\right)-\beta\left(f_{A}\right)$, where $\beta$ stands for the propagation constant. If the frequency difference between the two pumps is small, the term $\Delta \beta$ can be directly related to the group velocity dispersion by the expression $\Delta \beta$ $=\beta_{2}\left(f_{K}\right) \cdot\left(\omega_{K}-\omega_{I}\right)^{2}$, where $\beta_{2}$ is the second derivative of the propagation constant with respect to the frequency. Similar rules apply for the Stokes wave.

The basic idea of the local measurement of chromatic dispersion is to retrieve the power distribution of the Stokes or anti-Stokes wave along the fiber. With this power distribution and a simple time-frequency analysis, it is possible to retrieve the dispersion distribution along the fiber. A plain OTDR-like configuration for this measurement has been demonstrated [2]. Since the FWM conversion efficiency is small, very high FWM pump powers $(\sim 1 \mathrm{~W})$ are required. For 
these levels of pump power, modulation instability arises in the presence of small and positive dispersion. A different approach using drastically smaller FWM pump powers was proposed in [4]. It is based on performing Brillouin Optical Time Domain Analysis (BOTDA) over the FWM signal. Since stimulated Brillouin scattering can be made orders of magnitude more efficient than Rayleigh backscatter, smaller values of FWM pump powers are required.

For the BOTDA configuration, a spectrally narrow pump pulse is launched into the fiber in opposite direction. The optical frequency of the Brillouin pump pulse has to be approximately $f_{A}+v_{B}$ where $v_{B}$ is the Brillouin shift. For this configuration, the signal obtained at the BOTDA fiber end (tuned at $f_{A}$ ) can be expressed as:

$$
\Delta P_{A}=\frac{g(v)}{A_{e f f}} P_{A}(z) P_{P}(z) \Delta z
$$

where $g(v)$ is the Brillouin linear gain (dependent on the frequency separation between FWM and Brillouin pump), $A_{e f f}$ the mode effective area, $P_{A}$ and $P_{P}$ the powers of the anti-Stokes and Brillouin pump, respectively, and $\Delta z$ the pump pulse length. Since the Brillouin pump power is only subject to standard attenuation and thus varies very slowly, the amplified part of the signal $\Delta P_{A}(z)$ is essentially proportional to the local power $P_{A}(z)$ of the anti-Stokes signal. In conventional fibers, $g(v)$ has a narrow lorentzian shape with a maximum at $v_{B}(10.2-10.9 \mathrm{GHz}$ at $1550 \mathrm{~nm})$ and a width $\Delta v_{B}$ (between 30 and $50 \mathrm{MHz}$ at $1550 \mathrm{~nm}$ ). In the method reported in [4], the Brillouin pump was generated by a third laser, and thus keeping the three frequencies within $\Delta v_{B}$ was not stable for periods longer than a few minutes. In this paper, we present a significant improvement of this method, in which the Brillouin pump is generated by modulation and four-wave mixing, resulting in an ideally stable frequency difference between Brillouin pump and anti-Stokes FWM signal for any wavelength separation between FWM pumps. Thus, by varying $v$ it is possible to simultaneously retrieve the power distribution of the anti-Stokes signal and to measure the Brillouin shift. The maximum $v_{B}$ might vary along the fiber due to strain, temperature or doping concentration.

\section{EXPERIMENTAL SETUP AND RESULTS}

The experimental arrangement that we used is depicted in figure 1(a). In figure 1(b) we show the wavelength arrangement. Two tunable, distributed feedback lasers are used to generate the FWM pumps. The first pump is continuous-wave, while the second one is modulated, producing as a result two nearly symmetric sidebands separated approximately by the Brillouin shift. An electronic feedback control at the output of the electro-optical modulator ensures that no DC output goes through, even in the case of thermal changes in the device. One of the two sidebands is used (together with the first FWM pump) to generate the initial seed of the Brillouin pump pulse by four-wave mixing in a highly nonlinear semiconductor optical amplifier (SOA). The other sideband is used as a pump for the FWM process in the fiber. Thus, the frequency separation between the Brillouin pump and the FWM product generated in the fiber is constant (even in the case of random jitter in the frequency of the two DFB lasers) and tunable (by acting on the RF generator). The two FWM pumps are aligned and amplified to saturation before entering the fiber.

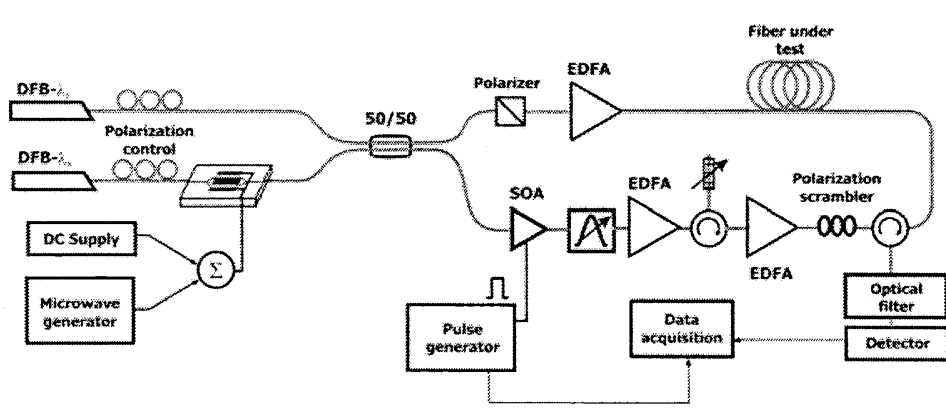

(a)

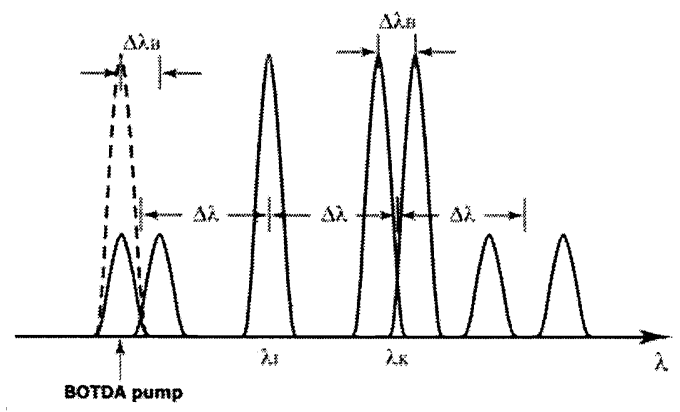

(b)

Fig. 1: (a) experimental arrangement and (b) wavelength arrangement. SOA: semiconductor optical amplifier, EDFA: erbium-doped fiber amplifier, BOTDA: Brillouin optical time domain analysis, EOM: electro-optical modulator. 
The Brillouin pump pulse is subsequently filtered (with a tunable thin film filter first, and a strain-tunable fiber Bragg grating later) and amplified with high-gain EDFAs, to rise its power from a few microwatts to a few hundred milliwatts. It should be noted that except the SOA gain bandwidth, all the rest of the instrument is basically tunable over a wide range of wavelengths. The Brillouin pump pulse is introduced in the fiber in counter propagating direction, thus producing local Brillouin gain of the FWM product along the fiber. This gain can be observed at the input end of the Brillouin pump pulse by a suitable detector and a digitizing oscilloscope, like in any other BOTDA setup. The polarization of the Brillouin pump pulse is scrambled, and the acquired traces are averaged several thousand times to ensure that no polarization-dependent gain is recorded in the trace. The frequency separation between the Brillouin pump pulse and the four-wave mixing is swept all over the region of Brillouin gain of the fiber. The final result is a 3D representation like the experimental results shown in figure 2.

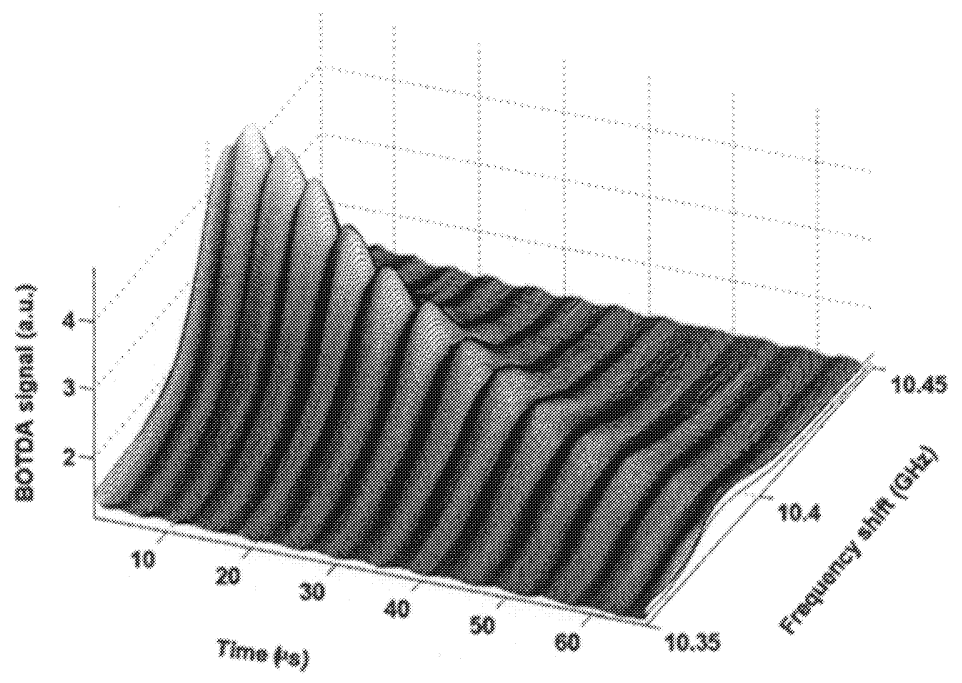

Fig.2: experimental trace obtained for a dispersion-shifted fiber. The average dispersion is approximately $1.1 \mathrm{ps} \cdot \mathrm{nm}^{-1} \cdot \mathrm{km}^{-1}$ and the wavelength separation between the FWM pumps is approximately $3.9 \mathrm{~nm}$.

The data is processed as follows: the Brillouin shift for each point is determined by use of a lorentzian shift. Once the Brillouin shift for each point is determined, we compute the power distribution of the anti-Stokes FWM signal. Over this power distribution along the fiber, we compute a spectrogram. The window of analysis gives the final resolution and uncertainty of the chromatic dispersion map. Bigger windows ensure less resolution but also a smaller uncertainty (bigger windows include more signal periods and thus a much more robust determination of the local oscillation). To figure out the precision of the local measurement, we have estimated that using a single period in the analysis yields an uncertainty in the frequency determination of approximately $\pm 5 \%$.

Figure 3(a) shows the final chromatic dispersion map together with the corresponding measured Brillouin shift distribution for a dispersion-shifted fiber. The experimental results are consistent, and in very good agreement with endto end measurements. Visually, a certain correlation between the evolution of the dispersion coefficient ( $D$ in the figure) and the Brillouin shift ( $B S$ in the figure) appears quite evident, although there seems to be no direct translation from one magnitude to the other. A model is being developed to explain this correlation, on the basis of doping concentration variation [5].

To verify this correlation, we performed the same measurements in dispersion-shifted fibers from other different manufacturers. Figure 3(b) shows the chromatic dispersion and Brillouin shift distribution along a fiber from manufacturer B. The correlation is less evident in this case, as in all the fibers measured from this manufacturer. The tested fiber spools from manufacturers $\mathrm{C}$ and $\mathrm{D}$ showed no significant chromatic dispersion variation, as well as no 
significant change in the Brillouin shift along the fiber. All the standard dispersion fibers tested (from all the manufacturers) showed no significant variation of the dispersion as well as rather negligible variations in the Brillouin shift.

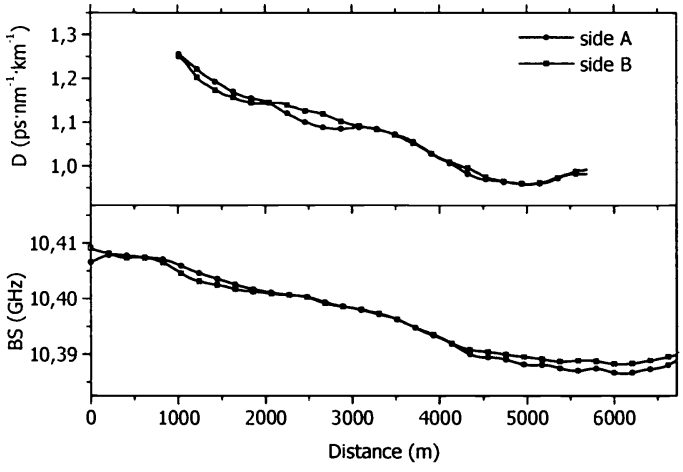

(a)

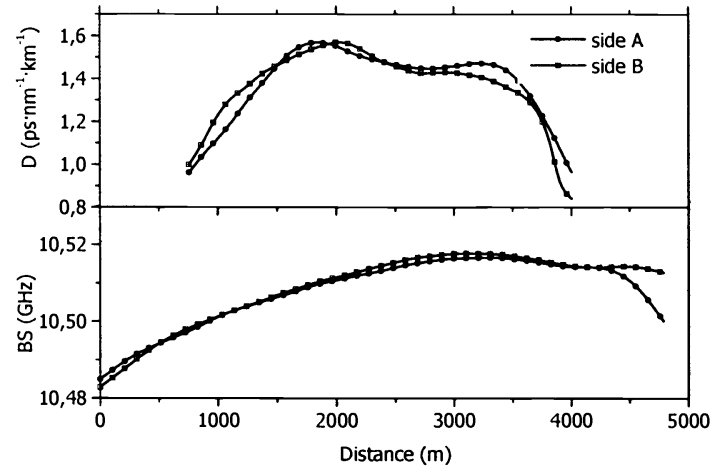

(b)

Fig. 3: experimental chromatic dispersion (labeled D) maps for both sides of two dispersion-shifted fibers of similar characteristics but different manufacturers together with the corresponding measurements of Brillouin shift distribution (labeled BS). The measures are performed from both ends of the fiber spools.

Regarding the dynamic range of the instrument, we have achieved successful mapping of $\sim 20-\mathrm{km}$ long concatenations of SMF fibers. We believe that a suitable improvement of some still unoptimised aspects of the system (namely increasing the trace averaging and reducing the detection bandwidth) would lead to a superior performance of the dynamic range. With these improvements, we expect to achieve a dynamic range similar to that of a conventional BOTDA setup, around $50 \mathrm{~km}$.

\section{CONCLUSION}

An experimental technique for the simultaneous measurement of local chromatic dispersion and Brillouin shift along an optical fiber has been demonstrated. The experimental results are consistent and in good agreement with end-to-end data. Preliminary results show a correlation between both quantities in certain kinds of fibers. Further work and modeling should provide more insight into this correlation.

\section{REFERENCES}

1. L. F. Mollenauer, P. V. Mamyshev, J. Gripp, M. J. Neubelt, N. Mamysheva, L. Gruner-Nielsen and T. Veng "Demonstration of massive wavelength-division multiplexing over transoceanic distances by use of dispersionmanaged solitons" Opt. Lett. 25, 704-706 (2000).

2. L. F. Mollenauer, P. V. Mamyshev and M. J. Neubelt, "Method for facile and accurate measurement of optical fiber dispersion maps" Opt. Lett. 21 1724-1726 (1996).

3. M. Eiselt, R. M. Jopson and R. H. Stolen, "Nondestructive position-resolved measurement of the zero-dispersion wavelength in an optical fiber" J. Lightwave Technol. 15 135-143 (1997)

4. M. Gonzalez-Herraez, L. Thevenaz and Ph. Robert "Distributed measurement of chromatic dispersion using fourwave mixing and Brillouin optical time domain analysis" Opt. Lett. 28 2210-2212 (2003).

5. M. Nikles, L. Thévenaz and Ph. Robert "Brillouin Gain Spectrum Characterization in single-mode optical fibers" J. Lightwave Technol. 15 1842-1851 (1997) 